\title{
PRIME: POWERLINE INTELLIGENT METERING EVOLUTION
}

\author{
Inigo BERGANZA \\ Iberdrola SA - Spain \\ iberganza@iberdrola.es
}

\author{
Alberto SENDIN \\ Iberdrola SA - Spain \\ asendin@iberdrola.es
}

\author{
Javier ARRIOLA \\ Iberdrola SA - Spain \\ fj.arriola@iberdrola.es
}

\begin{abstract}
Iberdrola, as part of a larger Consortium, started looking two years ago for a solution that could combine AMM deployment with SmartGrids needs. The output of this joint initiative is the PRIME Project.
\end{abstract}

\section{INTRODUCTION}

Whenever SmartGrids are discussed in Europe, it is apparent that advanced meter management (AMM) will be the first thing to happen in time. The European Directive 2006/32/EC on energy end-use efficiency and energy services supports the use of "intelligent metering systems such as individual metering instruments managed by remote" as a way to improve energy efficiency.

Now, more and more EU member states are implementing their own policies to ensure that customers will be able to fully benefit from current and future improvements in metering infrastructure and distribution grids. For Iberdrola in Spain, the Spanish Ministerial Order ITC/3860/2007 is a strong driver: it sets the obligation for all utilities to replace all their meters under $15 \mathrm{~kW}$ with new smart meters by 2018. This means the rollout of around 10 million units in the case of Iberdrola. In addition to economic and efficiency considerations, utilities will increasingly need to define their own strategies to fulfil the requirements for a new, costeffective metering solution that will be part of the SmartGrids of the future. Iberdrola has been carefully developing its strategy for two years now. All of its residential customers are currently being manually read six times per year, and the installed base is made up mostly of electromechanical meters. An obvious paradigm shift will be to consider meters as digital communication devices (nodes) which are part of a network; as such, metering will be just one of a variety of functions these smart meters will have. This communications network will be quite complex: different technologies will have to be integrated into it, wired and/or wireless. Power line communications (PLC) is a very strong proponent to become one of these technologies.

Iberdrola's extensive experience with BPL (broadband over power line) deployments over European grids since 2003 [1] and the direct involvement in EU projects like the Open PLC European Research Alliance (OPERA) have shown that the use of a utility's own distribution infrastructure is a clear advantage in terms of cost and efficiency.

In a meter market that will be open to competition, AMM rollouts will result in a non-homogenous meter infrastructure with different levels of functionalities within grid areas. So it seems mandatory to provide for a certain level of standardisation and interoperability between the meters to be installed. Iberdrola understands that current PLC-based AMM solutions lack the required level of standardisation and interoperability. Furthermore, most of the products in the market are proprietary, have been developed some time ago, and would imply additional risks on the utilities' investments, as their limited data rate and capabilities would not be able to support the expected functionalities of smart meters and SmartGrids. Thus, the PRIME initiative was started by Iberdrola back in December 2006 with the aim of promoting a Physical (PHY) and Medium Access Control (MAC) layer standard definition based on up-to-date technologies, in order to guarantee that future market requirements are met and that utility investments are safe.

\section{THE PRIME INITIATIVE}

The PRIME approach is straightforward: its target, once the viability of the idea has been assessed and proven right, is to define suitable specifications and test compliant systems in the field. PRIME is open, royalty and patent free, and it seeks interoperability for different vendors' equipment/systems as a means to push for the growth of a new market, so that ultimately all players network operators, retailers, manufacturers, and last but not least, the end user - will benefit.

Several entities have subsequently joined PRIME and current members include Advanced Digital Design, CURRENT Group, Landis+Gyr, STMicroelectronics, uSyscom and ZIV Medida. As can be seen, three different profiles of industry players were chosen: meter manufacturers that bring their know-how in metering, telecom service providers that bring fresh thinking and expertise in telecommunications, and semiconductor manufacturers that set up the limits of cost effectiveness to all. These companies have extensive experience in PLC and metering. Other utilities and companies have already expressed their interest in joining the project.

The PRIME timeline was defined as:

- Year 2007 - Concept testing (point to point), PHY and MAC specifications

- Year 2008 - Concept testing (point to multi-point) and prototype development - Field trials during Quarters 3 and 4

- Year 2009 - Large pilots, getting details and logistics ready

- Year 2010 - Deployment starts.

Thus the aim is that in 2010 Iberdrola and other utilities will start massive rollouts with PRIME-compliant systems.

Some field tests had already been performed during 2007: a measuring campaign including 200 sites from three different cities allowed further tuning of the details of the specifications. Milestones were fully achieved, and PRIME has defined comprehensive specifications for the PHY and MAC layers that form the basis for a low cost, 
high performance PLC system.

Even though extensive research and development is involved in the telecommunications area, the project was conceived not just as an R\&D project, but as an industrial project aimed at bringing to the marketplace interoperable products that are based on an open, public standard by 2010 .

\section{System architecture for AMM}

The PRIME system is composed of subnetworks, which are defined within the context of a transformer station and its meters. A subnetwork comprises a tree with two types of nodes, a base node and service nodes.

The base node is at the root of the tree and acts as the master node that provides connectivity to the subnetwork. It also manages the subnetwork resources and connections. There is only one base node in a subnetwork. This base node is initially the subnetwork itself, and the other nodes follow a process of registration in order to enrol themselves on this subnetwork.

The other nodes of the subnetwork are service nodes the leaves and branches of the tree. These nodes configure themselves to establish network connectivity up to the base node. Each of these nodes is one entity within the subnetwork. A terminal node is a leaf of the tree, while a switch node - a branch - is the basis for the connectivity within the subnetwork, by means of the repeating mechanisms implemented.

\section{PHY laver}

The PRIME PHY layer is designed to transmit and receive over power lines which were originally devised for distribution of power at $50-60 \mathrm{~Hz}$ AC. The use of this medium for communications at higher frequencies presents some technically challenging problems.

Distribution networks are usually made up of a random variety of conductor types, terminating into loads of different impedances. Such a network has an amplitude and phase response that varies widely with frequency. Furthermore, the channel characteristics can also vary with time as the loads on the network change.

Interference also affects power lines. Electric appliances with different kind of engines, switching power supplies and halogen lamps produce impulse noise that reduces the reliability of communication signals. Due to attenuation, the noise is also location dependent [2].

Thanks to a combination of approaches it is ultimately possible to overcome the hostility of the medium and allow for robust high speed, low cost communications over power lines. A simple yet powerful scheme has been devised, which is based on adaptively modulated Orthogonal Frequency Division Multiplexing (OFDM), along with forward error correction and data interleaving. In fact, one of the main novelties of PRIME is that it uses an OFDM approach instead of traditional single carrier solutions that have been used in the past for narrowband power line communications. OFDM is well known in the literature and in industry - it is currently used in xDSL technologies and terrestrial wireless distribution of television signals (DVB-T, DVB-H, etc.), and has also been adapted for IEEE's high rate wireless LAN standards (802.11a and 802.11g). In less than 20 years, in fact, OFDM has developed into a popular scheme for virtually all new telecoms standards: WiMAX, DAB,
DRM, 3G cellular telephony, UWB, MoCA, BPL, etc. [3] Furthermore, the OFDM concept is not completely new to PLC smart metering, as it is already supported by IEC 61334-5-4 under the name of MCM (multi-carrier modulation). The PRIME project will allow for real implementations based on evolution of this standard.

The primary advantage of OFDM is its ability to cope with severe channel conditions - attenuation of high frequencies in long power lines, narrowband interference and frequency selective fading due to multipath without complex additional mechanisms (e.g. equalisation filters). OFDM is also very robust against impulsive noise and it achieves high spectral efficiencies, thus allowing for higher data rates.

From the beginning, an important choice for PRIME was to select a frequency band that would:

- Allow inexpensive equipment implementations

- Have acceptable channel behaviour for transmission and reception over power lines, and

- $\quad$ Be future proof, i.e. that the frequency range should not be significantly impaired in the foreseeable future by other services (e.g. radio).

The natural choice for these needs was evident, as in Europe there is already a normalised frequency band for PLC, whose use "shall be restricted to electricity operators and their licensees". Known as the CENELECA band, this is defined in EN50065-1 and spans from 3 $\mathrm{kHz}$ to $95 \mathrm{kHz}$. In the US the FCC Part 15 allows the use of up to $500 \mathrm{kHz}$, so PRIME would also be compatible with this.

A total of 96 data subcarriers are transmitted between 42 $\mathrm{kHz}$ and $89 \mathrm{kHz}$, adaptively using one of three differential digital modulation schemes (BPSK, QPSK, 8PSK) and optionally a convolutional code. The number of subcarriers was seen to improve performance as it was increased, up to a certain limit which had to be kept for complexity reasons. The lower parts of CENELEC-A band are unused due to their inherent unreliability and noise levels.

Frequency domain differential PSK modulation was shown to be superior while allowing for low cost transceivers, and around one-third of measured cases correctly demodulated 8PSK, so it was decided to include non-binary constellations in the specification. This means PRIME will be able, depending on channel conditions, to achieve high speeds at the PHY level:

\begin{tabular}{|l|c|c|c|}
\hline & BPSK & QPSK & 8PSK \\
\hline Coding On & $21.4 \mathrm{kbps}$ & $42.9 \mathrm{kbps}$ & $64.3 \mathrm{kbps}$ \\
\hline Coding Off & $42.9 \mathrm{kbps}$ & $85.7 \mathrm{kbps}$ & $128.6 \mathrm{kbps}$ \\
\hline
\end{tabular}

These data rates are consistent with measurements taken in field trials during 2007.

All technical decisions made and included in the PHY specification are the result of the analysis of several options that were preliminarily implemented and tested in the field. Thus, the final picture is the one that offered the best compromise in terms of performance, flexibility and cost for the overwhelming majority of measurements.

\section{MAC layer}

The MAC layer is defined for a connection oriented master/slave configuration, and it is optimised for a low voltage power line environment. Well known procedures 
and mechanisms from other public standards, and common practices have been included.

A specific addressing scheme was defined to identify subnetworks and different types of nodes, manage connections, and perform multicast and broadcast. Connections are needed for communication of nodes in the system. These connections have different scopes, basically local and global, defining different end-to-end levels of communication.

PRIME devices will mostly be used in applications which generate small or very small bursts of traffic and may have real time delay requirements. For this reason, the channel access scheme is based mainly on CSMA/CA (carrier sense multiple access with collision avoidance), although it is possible to reserve contention-free periods for certain types of traffic. Time is divided into fixed size frames, and transmission of a beacon by a base node identifies the start of a frame.

All network elements are able to configure themselves automatically, responding to changing channel conditions. These same elements are able to remain connected to their subnetwork, thanks to the use of several ad-hoc mechanisms.

A repeating function is defined as an integral part of the MAC layer for purposes of scalability and range extension. The switch nodes perform the repeating function, which includes:

- Transmission beacons at regular intervals so that new terminal nodes can synchronise to the network and existing terminal and switch nodes remain synchronised

- $\quad$ Relaying data and control packets to/from nodes in their domain from/to the base node.

Additionally, a selective hop-by-hop automatic repeat request (ARQ) mechanism is used for error control so that the MAC layer can ask for PHY retransmission whenever it is needed.

With regard to security, PRIME is currently using flexible key management policies and 128 bit AES (advanced encryption standard) to provide privacy, authentication and data integrity. Both data and control messages are encrypted in the system.

Using PRIME MAC with already existing upper layers is a critical issue, given the fact that bandwidth is a scarce resource. Several convergence layers are being devised to adapt the system to different applications: they all have a minimum overhead and take the most out of the capabilities of the PRIME MAC layer. Apart from performance issues, PRIME faces the challenge of interfacing with common applications and technologies such as IP, metering and remote control applications, and others.

\section{THE FUTURE}

So far, the PHY and MAC layers have been thoroughly discussed and partially tested in a variety of low voltage networks. Based on the results, the best options at PHY and MAC levels have been included in the specifications. Overall, the performance of the PRIME system in the field has been better than expected.

Minor technical details will be further tuned during 2008 to be included in the final version of the specifications. PRIME members are now confident the objectives defined at the beginning of the process will be cost effectively met. Currently three different manufacturers are developing PRIME-compliant systems so that at the end of 2008 there will be a real AMM solution that has demonstrated interoperability and full scalability for mass deployments.

Such a system could be the basis of an open, public standard for high speed communications over power lines that would perfectly fit the needs of utilities developing AMM and smart grids. PRIME partners are fully convinced that openness of this future-proof solution will be rewarded by the market above all other proprietary systems.

A detailed White Paper giving some insights on $\mathrm{PHY}$ layer is downloadable for free at http://www.iberdrola.com/suppliers/SmartMetering The 47 page PHY specification itself is available at AMI.info@iberdrola.es

\section{REFERENCES}

[1] A. Sendin, 2004, "PLC Commercial Deployment of Iberdrola”, International Symposium on Power Line Communications and Its Applications, 2004.

[2] M. Zimmermann and K. Dostert, 2002, "Analysis and modelling of impulsive noise in broad-band power line communications", IEEE Transactions on Electromagnetic Compatibility, vol. 44, no. 1, pp. 249-258.

[3] J. A. C. Bingham, 1990, "Multicarrier modulation for data transmission: An idea whose time has come," IEEE Communications Magazine, vol. 28, no. 5, pp. 5-14. 